\title{
Dysregulated plasma levels of miRNA-132 and miRNA-134 in patients with obsessive-compulsive disorder
}

\author{
Jihui Yue ${ }^{1,2}$, Baoli Zhang ${ }^{3}$, Hong Wang ${ }^{1}$, Xuejiao Hou ${ }^{1}$, Xingyu Chen ${ }^{1}$, Minfeng Cheng ${ }^{1}$, Shenglin Wen ${ }^{1,2}$ \\ ${ }^{1}$ Department of Psychiatry, Fifth Affiliated Hospital, Sun Yat-sen University, Zhuhai, China; ${ }^{2}$ Guangdong Provincial Key Laboratory of Biomedical \\ Imaging and Guangdong Provincial Engineering Research Center of Molecular Imaging, Fifth Affiliated Hospital, Sun Yat-sen University, Zhuhai, \\ China; ${ }^{3}$ Mental Health Center, Renmin Hospital of Wuhan University, Wuhan, China \\ Contributions: (I) Conception and design: S Wen, M Cheng, J Yue; (II) Administrative support: S Wen; (III) Provision of study materials or patients: B \\ Zhang; (IV) Collection and assembly of data: M Cheng, B Zhang; (V) Data analysis and interpretation: J Yue; (VI) Manuscript writing: All authors; (VII) \\ Final approval of manuscript: All authors. \\ Correspondence to: Prof. Shenglin Wen; Minfeng Cheng. Department of Psychology, Fifth Affiliated Hospital, Sun Yat-sen University, No. 52 Meihua \\ East Road, Zhuhai, China. Email: wenshl@mail.sysu.edu.cn; Emin7641@163.com.
}

\begin{abstract}
Background: Obsessive-compulsive disorder (OCD) is a severe, chronic, disabling neuropsychiatric disorder, the pathophysiology of which has yet to be fully understood. In this study, we aimed to detect the levels of miRNA-132 and miRNA-134 in the plasma of patients with OCD and to analyze the factors influencing OCD.

Methods: The levels of miRNA-132 and miRNA-134 in peripheral blood of 30 patients with OCD and 32 normal controls were detected by quantitative reverse transcription polymerase chain reaction (RT-qPCR). Patients were assessed using clinical scales, including the Hamilton Anxiety Rating Scale (HAMA), Hamilton Depression Rating Scale (HAMD), and Yale-Brown Obsessive-Compulsive Scale (Y-BOCS).

Results: The plasma levels of miRNA-132 and miRNA 134 in the OCD group were significantly higher than those in the control group $(\mathrm{P}<0.05)$. There was no significant correlation between the plasma levels of miR-132 and miR-134 in the OCD group and general demographic (gender, age, and education level) and clinical characteristics (duration of disease, HAMA, HAMD, and Y-BOCS scores).

Conclusions: Patients with OCD have abnormal plasma levels of miRNA-132 and miRNA-134, which may influence the number of dendrites in the cerebral cortex and formation of synapses. Therefore, miRNA-132 and miRNA-134 plasma levels should be considered as potential biomarkers for OCD detection.
\end{abstract}

Keywords: Obsessive-compulsive disorder (OCD); miRNA-132; miRNA-134; synaptic plasticity

Submitted Jun 14, 2020. Accepted for publication Aug 06, 2020.

doi: $10.21037 /$ atm-20-5217

View this article at: http://dx.doi.org/10.21037/atm-20-5217

\section{Introduction}

Obsessive-compulsive disorder (OCD) is a severe, chronic, and disabling neuropsychiatric disorder. OCD is a complex disorder that is influenced by gene-environment interactions $(1,2)$. Previous studies have confirmed significant familial aggregation of OCD $(2,3)$, and multiple susceptibility genes $(4,5)$ and neuroplasticity (6) have been associated with its pathogenesis. Despite clinical trials of selective serotonin reuptake inhibitors (SSRIs) and cognitive-behavioral therapy producing adequate results, residual symptoms were still reported in $40-60 \%$ of patients, which stresses the need for augmentation strategies to be developed $(7,8)$. In addition to the serotonergic and dopaminergic systems, studies have also identified other genes, such as SAPAP/ DLGAP, SLC1A1, GRIN /GRIK, BDNF, and NGFR/ NTRK2, as contributing to the increased risk of $\operatorname{OCD}(4,9)$.

MicroRNAs (miRNAs) are a highly conserved 
group of small, non-coding RNA molecules, which are 19-25 nucleotides in size and are believed to control the activity of as many as $30 \%$ of all protein-coding genes in mammals (10). MiRNAs act as fine tuners and on-off switches in gene expression patterns, playing a pivotal role in synaptic plasticity in the central nervous system (CNS) (6). MiR-132, for instance, is closely associated with neurite outgrowth, while miR-134 serves a significant role in postsynaptic regulation; this suggests that brain-specific miRNA families are crucial for synaptic plasticity. Brainderived neurotrophic factor (BDNF) and cAMP-response element-binding protein (CREB) are two closely related genes that are substantially involved in synaptic plasticity, as well as the modulation of synapse formation (11). There is an evidence that BDNF and CREB may mediate the occurrence of OCD $(12,13)$.

MicroRNA-132 (miR-132) is significant for regulating neuronal differentiation, maturation, and functioning, and participates broadly in axon growth, neural migration, and plasticity $(14,15)$. BDNF upregulates CREB by acting on neurons, thus promoting the expression of miR-132. miR132 was revealed in a previous study to influence neurodevelopment by regulating the expression level of methyl-CpG binding protein 2 (MeCP2) (16). miR132 inhibits the expression of $\mathrm{MeCP} 2$ by targeting the three prime untranslated region (3'-UTR) of MeCP2 mRNA, while MeCP2 increases the content of BDNF, which regulates the expression of the miR-132 cluster, thereby maintaining the stability of the brain environment. Changes in the level of miR-132 in the brain affect the secretion of $\mathrm{MeCP} 2$, and an imbalance in the level of MeCP2 can lead to nervous system disorders, such as Rett syndrome.

MicroRNA-134 (miR-134) plays a significant role in synaptic plasticity and modulating synapse formation. MiR134 , which is localized to the synaptodendritic compartment of rat hippocampal neurons, negatively regulates the size of dendritic spines, which are postsynaptic sites of excitatory synaptic transmission (17). Besides, miR-134-5p inhibits the translation of LIM domain kinase 1 (Limk1) at the synapse until synaptic activation, at which point miR-134$5 \mathrm{p}$ is inactivated and Limk1 protein is expressed, leading to dendritic spine growth (18). Moreover, BDNF could relieve the repressive effect of miR-134 on Limk1 mRNA translation. The regulation of translation in dendrites by microRNAs, in a BDNF-sensitive manner, may constitute a mechanism whereby the neurotrophin regulates the postsynaptic proteome during the late phases of longterm potentiation (LTP). Another study demonstrated that
miR-134 regulates memory and neuronal plasticity (19). Furthermore, Sirtuin 1 (SIRT1) normally functions to limit miR-134 expression via a repressor complex containing transcription factor Yin Yang 1 (YY1). Unchecked miR-134 expression after SIRT1 deficiency leads to down-regulated expression of CREB and BDNF, thereby impairing synaptic plasticity.

Although There are several studies reporting the role of miRNAs in OCD (20), such as miR-485, miR-488, the study about miRNAs is still much few. Studies found that miR-132 and miR-134 are related to BDNF; the level of BDNF is regulated by miR-132 and miR-134, while the levels of miR-132 and miR-134 are influenced by BDNF $(16,19)$. As BDNF levels are correlated with OCD, we speculate the plasma levels of miR-132 and miR-134 may also be related to OCD; however, no studies to date have examined this possible relationship. Hence, the present study aimed to analyze the plasma levels of miRNA-132 and miRNA-134 in patients with OCD and to assess the factors influencing OCD.

We present the following article in accordance with the MDAR reporting checklist (available at http://dx.doi. org/10.21037/atm-20-5217).

\section{Methods}

\section{Participants}

Between July, 2014, and February, 2016, study subjects were recruited from the Third Affiliated Hospital of Sun Yatsen University (Guangzhou, China). The subjects had been diagnosed with OCD by two psychiatrists. The control group comprised employees, students, and patients from the Third Affiliated Hospital of Sun Yat-sen University who met the inclusion and exclusion criteria, and were identified via health checkups. A signed informed consent form was obtained from each of the study subjects or their legal guardians. The study was approved by the Ethics Committee of the Third Affiliated Hospital of Sun Yatsen University and was conducted in accordance with the Declaration of Helsinki (as revised in 2013).

The inclusion criteria for subjects were as follows: (I) meeting the criteria of the Diagnostic and Statistical Manual of Mental Disorders, fifth edition (DSM-5) presented by the American Psychiatric Association (Washington, D.C., USA); (II) aged between 16 and 50 years old; (III) a YaleBrown Obsessive-Compulsive Scale (Y-BOCS) score $>16$ points; for simple obsessive or forced behavior, a Y-BOCS 
Table 1 Demographic and clinical characteristics of OCD patients and healthy controls

\begin{tabular}{|c|c|c|c|c|}
\hline Variable & OCD group & Control group & $\mathrm{t} / \chi^{2} / Z$ & $\mathrm{P}$ \\
\hline Male & $17(65.4 \%)$ & $12(42.9 \%)$ & & \\
\hline Female & $9(34.6 \%)$ & $16(57.1 \%)$ & & \\
\hline Age (years) & $24.19 \pm 7.67$ & $26.14 \pm 9.67$ & 0.817 & 0.418 \\
\hline Duration of illness (years) & $3.34 \pm 2.61$ & & & \\
\hline Y-BOCS score & $24.15 \pm 6.14$ & & & \\
\hline HAMD score & $11.00 \pm 4.38$ & & & \\
\hline HAMA score & $6.19 \pm 3.35$ & & & \\
\hline
\end{tabular}

$\mathrm{P}<0.05$ denotes statistical significance. OCD, obsessive-compulsive disorder; Y-BOCS, Yale-Brown Obsessive-Compulsive Scale; HAMD, Hamilton Depression Rating Scale; HAMA, Hamilton Anxiety Rating Scale.

score $>8$ points; (IV) no other major mental illnesses in the first 3 months after enrollment; (V) no psychiatric drug use; (VI) discontinuing medication for more than 5 half-lives; and (VII) signed the written informed consent form.

The exclusion criteria were as follows: (I) a history of organic brain disease or serious physical illness, symptoms of psychosis, or a history of other mental illnesses; (II) severe depression (a Hamilton Depression Rating Scale (HAMD) score $>35$ points); or (III) second-generation with a family history of mental illness.

As shown in Table 1, a total of 26 patients (17 males and 9 females), aged between 16 and 49 (median, $24.19 \pm 7.67)$ years old, were enrolled. Disease duration ranged from 9 months to 10 years (median duration, 3.49 years). The control group comprised 28 individuals (12 males and 16 females), aged between 18 and 45 (median, $26.14 \pm 9.67)$ years old. There were no significant differences between the OCD group and the control group in terms of gender $\left(\chi^{2}=2.752, \mathrm{P}=0.097\right)$ and age $(\mathrm{t}=0.817, \mathrm{P}=0.418)$.

\section{Detection of plasma levels of miRNA-132 and miRNA-134}

A venous blood sample $(4 \mathrm{~mL})$ was collected from each study subject in ethylenediaminetetraacetic acid (EDTA) tubes. After centrifugation at $4{ }^{\circ} \mathrm{C}$ for $10 \mathrm{~min}$ at $2,400 \mathrm{rpm}$, $1.5 \mathrm{~mL}$ of the upper layer of plasma was taken and stored at $-80{ }^{\circ} \mathrm{C}$ for examination. Quantitative reverse transcription polymerase chain reaction (RT-qPCR) was carried out to detect miRNA-132 and miRNA-134, with miRNA-16 used as an internal control. Total RNA was extracted using the miRVana PARIS kit (AM1556; Life Technologies, Carlsbad, CA, USA), and reverse transcription was performed with the Transcriptor First Strand cDNA Synthesis kit (Roche, Basel, Switzerland). FastStart Universal SYBR Green Master (ROX; Roche, Basel, Switzerland) was used for fluorescence quantification.

The process was conducted as follows

(I) Extraction of total RNA:

(i) Plasma $(1 \mathrm{~mL})$ was taken and placed in a $1.5 \mathrm{~mL}(500 \mu \mathrm{L}$ each) Eppendorf tube (Hamburg, Germany).

(ii) Next, $200 \mu \mathrm{L}$ per well of fixing/denaturing solution was added to each tube, along with $700 \mu \mathrm{L}$ of Acid-Phenol Chloroform. The plasma was then centrifuged at $10,000 \mathrm{~g}$ for $5 \mathrm{~min}$ at room temperature.

(iii) The supernatant was carefully transferred into a new $2.0 \mathrm{~mL}$ Eppendorf tube, and $10 \mathrm{~g}$ of sodium acetate was added (anhydrous; $0.24 \mathrm{M}$ ).

(iv) The cleaned spin column was placed into a clean collection tube, and the mixture from the previous step was piped ( $700 \mu \mathrm{L}$ each time) to the spin column and centrifuged at $6,000 \mathrm{~g}$ for $30 \mathrm{~s}$ at room temperature. The filter solution was discarded, and the spin column was repositioned into the collection tube. This step was repeated 
until all the liquid had passed through the column.

(v) A pipette was used to put $700 \mu \mathrm{L}$ of wash solution 1 into a spin column. After centrifugation at $6,000 \mathrm{~g}$ for $30 \mathrm{~s}$ at room temperature, the filter solution was discarded, and the spin column was repositioned into the collection tube.

(vi) The spin column was washed twice with $500 \mu \mathrm{L}$ of phosphate-buffered saline (PBS) solution.

(vii) The spin column was emptied for $1 \mathrm{~min}$ and then placed in a new collection tube. After that, $35 \mu \mathrm{L}$ of $95^{\circ} \mathrm{C}$ preheated elution solution was added to the center of the column and centrifuged at $10,000 \mathrm{~g}$ for $30 \mathrm{~s}$ at room temperature. The liquid in the collection tube was discarded and stored at $-80^{\circ} \mathrm{C}$.

(II) Preparation of cDNA:

(i) Total RNA $(10 \mu \mathrm{L})$ was placed in a $200 \mu \mathrm{L}$ RNAse-Free EP tube.

(ii) Next, $3 \mu \mathrm{L}$ of RT primer working solution was added and mixed for $15 \mathrm{~s}$.

(iii) The solution was incubated on an ice bath at $370{ }^{\circ} \mathrm{C}$ for $10 \mathrm{~min}$.

(iv) Then, $2 \mu \mathrm{L} \mathrm{dNTP}(10 \mu \mathrm{mol} / \mathrm{L})$ was added and mixed.

(v) RNase inhibitor $(0.5 \mu \mathrm{L})$ was added, and reverse transcription was performed using $0.5 \mu \mathrm{L}$ ReverseTra Ace, before mixing and centrifugation.

(vi) The solution was incubated at $25^{\circ} \mathrm{C}$ for $10 \mathrm{~min}$, at $55^{\circ} \mathrm{C}$ for $60 \mathrm{~min}$, at $85^{\circ} \mathrm{C}$ for $5 \mathrm{~min}$ to extinguish the reverse transcriptase.

(vii) The cDNA solution was frozen at $-20^{\circ} \mathrm{C}$.

(III) RT-qPCR

RT-qPCR was carried out on an ABI 7500 Real-Time PCR System (Bio-Rad Laboratories, Inc., Hercules, CA, USA) using the SYBR Green I dye method for detection and analysis. miRNA-16 was used as an internal control. The reaction conditions were set to pre-denaturation at $95{ }^{\circ} \mathrm{C}$ for $10 \mathrm{~min}$, denaturation at $95{ }^{\circ} \mathrm{C}$ for $15 \mathrm{~s}$, and annealing at $60{ }^{\circ} \mathrm{C}$ for $32 \mathrm{~s}$. The relative miRNA expression levels were calculated using the $2^{-\Delta \Delta \mathrm{Ct}}$ method.

\section{Evaluation of symptoms of psychosis}

On the first day of admission, the investigators assessed the subjects' obsessive-compulsive symptoms and emotional symptoms using the Y-BOCS, Hamilton Anxiety Rating Scale (HAMA), and HAMD.

Initially presented by the Goodman et al. in 1989 ,
Y-BOCS is used to assess the severity of symptoms for OCD patients around the world. The scale comprises 10 items: the first 5 items reflect forced thinking, and the second 5 items indicate forced behavior. The severity of symptoms of OCD are scored using a 5-point scale (0-4 points). The higher the score, the more severe the disease. Y-BOCS classifies OCD into different levels of severity: subclinical ( $0-7$ points), mild ( $8-15$ points), moderate (16-23 points), severe (24-31 points), and extremely severe (32-40 points). Additionally, according to the patients' scores on the forced-thinking and the forcedbehavior subscales, they can divided into different levels of severity: mild ( $4-7$ points) moderate ( $8-11$ points), severe (12-15 points), and very severe (16-20 points).

HAMA and HAMD scores are assessed using 14 HAMDs and 14 HAMDs to establish if a patient has major depressive disorder. HAMD $<8$ points indicates no depressive symptoms; HAMD $>21$ points shows mild or moderate depression; and HAMD $>35$ points indicates severe depression. HAMA $<7$ points indicates no symptoms of anxiety; 7-14 indicates the possibility of anxiety; HAMA $>14$ points indicates the existence of anxiety; HAMA in the range of 21-29 points indicates obvious anxiety; and HAMA $>29$ indicates severe anxiety.

\section{Statistical analysis}

Statistical analysis was performed using SPSS 19.0 software (IBM, Armonk, NY, USA). As the plasma levels of miR132 and miR-134 in the OCD group and the control group failed to meet the requirements of the $t$-test (normal distribution and variance), the two groups were compared using the rank-sum test. Spearman's correlation analysis was employed to analyze the relationship between the plasma levels of miRNA-132 and miRNA-134 and clinical features. $\mathrm{P}<0.05$ was considered to be statistically significant.

\section{Results}

\section{Comparison of plasma levels of miR-132 and miR-134 between the OCD group and the control group}

As shown in Table 2, the plasma levels of miR-132 and miR-134 in the OCD group were significantly higher than those in the control group. The plasma levels of miR-132 and miR-134 in the two groups were compared using the rank-sum test. The miR-132 level in the OCD group was markedly higher than that in the control group $(\mathrm{Z}=2.285$, 
$\mathrm{P}=0.022$ ). Similarly, the level of miR-134 in the OCD group was significantly higher than that in the control group $(\mathrm{Z}=2.122, \mathrm{P}=0.035)$.

\section{Correlation analysis of miR-132 and miR-134 levels in the OCD group and clinical indicators}

Figure 1 shows that the levels of miR-132 and miR-134 were not normally distributed. As shown in Table 3, spearman's

Table 2 Comparing plasma levels of miR-132 and miR-134 between the OCD group and the control group

\begin{tabular}{lcccc}
\hline \multirow{2}{*}{ Items } & \multicolumn{3}{c}{ Groups } & \multirow{2}{*}{$Z$} \\
\cline { 2 - 3 } & OCD group & Control group & \\
\hline miR-132 & 32.58 & 22.79 & 2.285 & 0.022 \\
miR-134 & 32.19 & 23.14 & 2.122 & 0.035 \\
\hline
\end{tabular}

$\mathrm{P}<0.05$ denotes statistical significance. OCD, obsessivecompulsive disorder. correlation analysis revealed that the levels of miR-132 and miR-134 were not significantly correlated with disease course, HAMA, HAMD, Y-BOCS scores, forced thinking, or forced behavior.

Correlation analysis of the levels of miR-132 and miR-134 in the OCD group and general demographic and clinical features

As presented in Table 4, the levels of miR-132 and miR134 did not follow normal distribution or homogeneity of variance. Consequently, the Wilcoxon rank-sum test for two independent samples was used. The results showed no correlation between gender and the levels of miR-132 and miR-134 (W=92, $\mathrm{P}=0.120 ; \mathrm{W}=99, \mathrm{P}=0.241)$, and the difference between the levels of miR-132 and miR-134 at different ages was not statistically significant $(W=126$, $\mathrm{P}=0.660 ; \mathrm{W}=115, \mathrm{P}=0.310$ ). Similarly, the course of the disease had no noticeable correlation with the levels of miR132 and miR-134 ( $\mathrm{W}=127, \mathrm{P}=0.698 ; \mathrm{W}=114, \mathrm{P}=0.286)$.
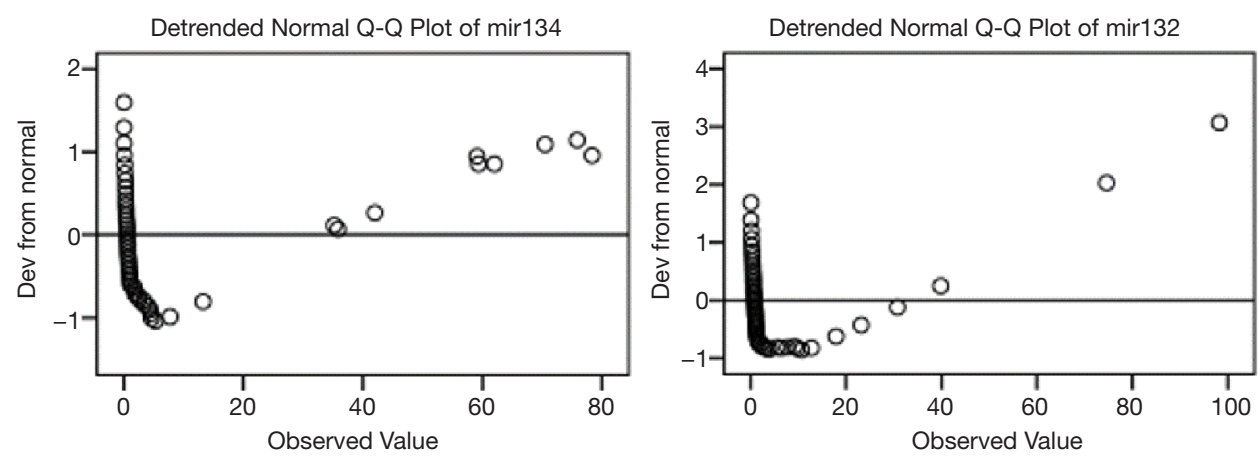

Figure 1 The levels of miR-132 and miR-134 did not obey normal distribution and homogeneity of variance.

Table 3 Correlation analysis of miR-132 and miR-134 in the OCD group and clinical indicators

\begin{tabular}{lcccc}
\hline \multirow{2}{*}{ Variable } & \multicolumn{2}{c}{ mir-132 } & & mir-134 \\
\cline { 2 - 5 } & $\mathrm{R}$ & $\mathrm{P}$ & $\mathrm{R}$ & $\mathrm{P}$ \\
\hline Duration of illness & -0.179 & 0.382 & -0.315 & 0.116 \\
HAMA & 0.205 & 0.316 & 0.282 & 0.163 \\
HAMD & 0.012 & 0.952 & 0.141 & 0.243 \\
Y-BOCS & -0.178 & 0.383 & 0.058 & 0.793 \\
OS & -0.202 & 0.323 & 0.212 & 0.297 \\
CS & -0.134 & 0.514 & 0.05 & \\
\hline
\end{tabular}

$\mathrm{P}<0.05$ denotes statistical significance. OCD, obsessive-compulsive disorder; HAMD, Hamilton Depression Rating Scale; HAMA, Hamilton Anxiety Rating Scale; Y-BOCS, Yale-Brown Obsessive-Compulsive Scale; OS, obsessive symptom; CS, compulsive symptom 
Table 4 Correlation analysis of the levels of miR-132 and miR-134 in the OCD group and general demographic and clinical features

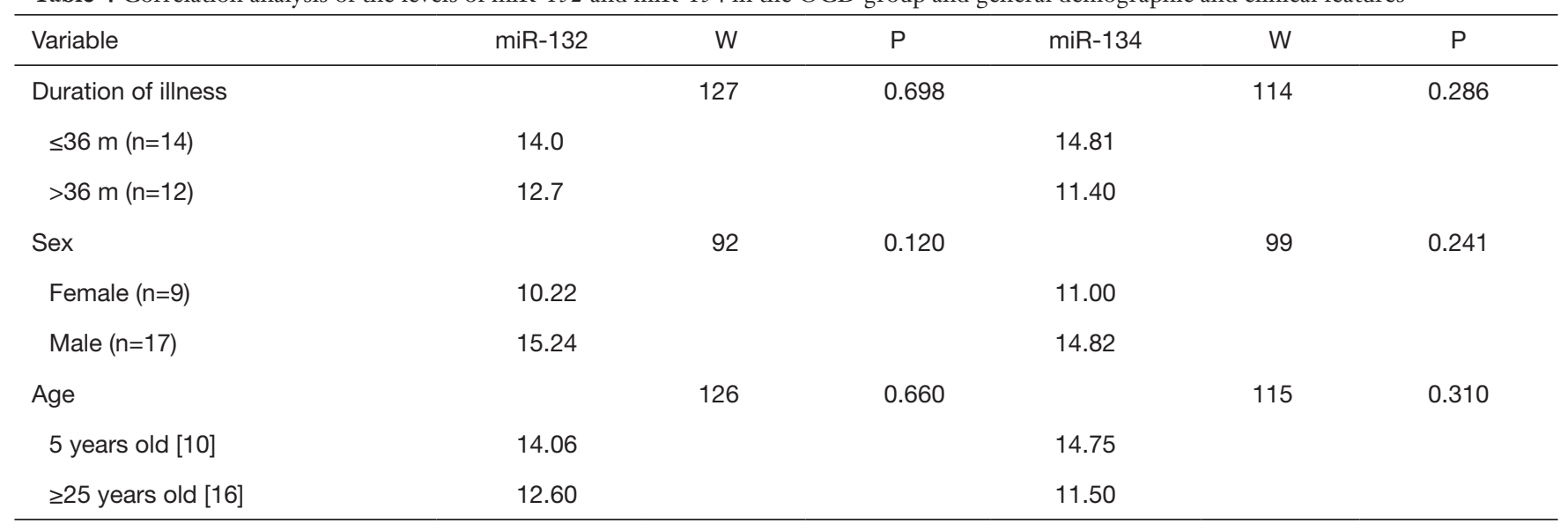

$\mathrm{P}<0.05$ denotes statistical significance. OCD, obsessive-compulsive disorder.

\section{Discussion}

Several studies have reported that synaptic plasticity plays a pivotal role in mental disorders (21-23), including OCD $(3,6)$. miRNAs act as fine tuners and on-off switches in gene expression patterns, especially in synaptic plasticity $(6,10,11,17,18)$. MiR-132 and miR-134 are members of the miRNA family and play a significant role in the regulation of synaptic plasticity in neuropsychiatric disorders $(14,18,19,24,25)$.

MiR-132 is an endogenous small RNA that controls posttranscriptional regulation of gene expression via controlled mRNA degradation or transcription inhibition. MiR132 is influential in the pathogenesis of various diseases, particularly those related to the CNS. The dysregulation of miR-132 results in the occurrence and exacerbation of neural developmental, degenerative diseases [such as Alzheimer's disease (AD), Parkinson's disease (PD), and epilepsy], neurological infections, and psychiatric disorders (14). Analysis of a PD rat model revealed that the expression level of BDNF was reduced in the midbrain and plasma, whereas miR-132 expression was significantly increased (26). Nudelman $e t a l$. found that pilocarpine-induced epilepsy led to a significant upregulation of pre-miR-132 and mature miR-132 (27). Studies on the functional sequence of miR132 demonstrated that by inhibiting miR-132 expression, hippocampal neuron damage can be remarkably attenuated, confirming that the overexpression of miR-132 aggravates neuronal damage (28). This study discovered that the plasma miR-132 level in the OCD group was markedly higher than that in the control group. We hypothesized that the upregulation of miR132 may directly or indirectly affect neural signaling to some extent, thereby resulting in excessive axonal damage. However, to confirm this hypothesis, further research needs to be carried out.

MiR-134 also plays a substantial role in synaptic plasticity and modulation of synapse formation. One previous study demonstrated the neuroprotective effect of a miR-134 antagomir, which can reduce ischemic injury and lead to prolonged seizure suppression (17). The current study found that the plasma miR-134 level in the OCD group was significantly higher than that in the control group. In neurons, miR-134 expression restricts excitatory synapse development, whereas its inhibition promotes dendritic spine growth (11). Gao et al.'s study showed miR-134 to be involved in SIRT1 modulation of synaptic plasticity and memory formation (19). They found that SIRT1 functions to limit miR-134 expression through a repressor complex containing transcription factor YY1, and unchecked miR134 expression after SIRT1 deficiency results in the downregulation of CREB and BDNF expression, which impairs synaptic plasticity.

The results of this study indicated that there was no correlation between the plasma levels of miR-132 and miR134 in the OCD group. Moreover, gender, age, disease duration, HAMD score, HAMA score, and Y-BOCS score, as well as elevated plasma levels of miR-132 and mrR134 in patients with first-onset OCD may be independent of the clinical symptoms.

The important role of miR-132 and miR-134 in neuroplasticity prompted us to explore their function in the pathogenesis of OCD. These miRNAs offer great potential as biomarkers for OCD detection because of 
their remarkable stability in blood and their characteristic expression in various diseases $(11,13)$.

The current cross-sectional case-control study has some limitations. First, there is a lack of comparison before and after treatment. Further, the plasma levels of miR-132 and miR-134 were detected by RT-qPCR, which is less sensitive than the TaqMan probe method. Another, in this study, the sample size is insufficient, which is the deficiency of this study, so we will further expand the sample size to do more in-depth research in the next step. Hence, a multi-center, prospective study is necessary to verify our findings and to address the above-mentioned deficiencies.

\section{Conclusions}

Patients with OCD have abnormal plasma levels of miRNA-132 and miRNA-134, which may influence the number of dendrites in the cerebral cortex and synapse formation. Therefore, miRNA-132 and miRNA-134 should be considered as biomarkers for OCD detection.

\section{Acknowledgments}

We would like to thank all of the individuals who participated in this study. Wen Shenglin designed the study. Yue Jihui and Zhang Baoli collected the original data and wrote the manuscript. Zhang Baoli completed the experimental analysis. Cheng Minfeng and Wang Hong managed and analyzed the data. Chen Xingyu was responsible for literature review. All the authors read and approved the final version of the manuscript.

Funding: This work was supported by the Technology Project of Guangdong Province, China (grant number 2014A020212530).

\section{Footnote}

Reporting Checklist: The authors have completed the MDAR reporting checklist. Available at http://dx.doi.org/10.21037/ atm-20-5217

Data Sharing Statement: Available at http://dx.doi. org/10.21037/atm-20-5217

Conflicts of Interest: All authors have completed the ICMJE uniform disclosure form (available at http://dx.doi. org/10.21037/atm-20-5217). The authors have no conflicts of interest to declare.
Ethical Statement: The authors are accountable for all aspects of the work in ensuring that questions related to the accuracy or integrity of any part of the work are appropriately investigated and resolved. A signed informed consent form was obtained from each of the study subjects or their legal guardians. The study was approved by the Ethics Committee of the Third Affiliated Hospital of Sun Yat-sen University (No. ChiCTR-CCS-12002164) and was conducted in accordance with the Declaration of Helsinki (as revised in 2013).

Open Access Statement: This is an Open Access article distributed in accordance with the Creative Commons Attribution-NonCommercial-NoDerivs 4.0 International License (CC BY-NC-ND 4.0), which permits the noncommercial replication and distribution of the article with the strict proviso that no changes or edits are made and the original work is properly cited (including links to both the formal publication through the relevant DOI and the license). See: https://creativecommons.org/licenses/by-nc-nd/4.0/.

\section{References}

1. Murphy DL, Moya PR, Fox MA, et al. Anxiety and affective disorder comorbidity related to serotonin and other neurotransmitter systems: obsessive-compulsive disorder as an example of overlapping clinical and genetic heterogeneity. Philos Trans R Soc Lond B Biol Sci 2013;368:20120435.

2. Nestadt G, Samuels J, Riddle M, et al. A family study of obsessive-compulsive disorder. Arch Gen Psychiatry 2000;57:358-63.

3. Taylor S. Etiology of obsessions and compulsions: a metaanalysis and narrative review of twin studies. Clin Psychol Rev 2011;31:1361-72.

4. Taylor S. Molecular genetics of obsessive-compulsive disorder: a comprehensive meta-analysis of genetic association studies. Mol Psychiatry 2013;18:799-805.

5. Cross-Disorder Group of the Psychiatric Genomics Consortium. Genomic Relationships, Novel Loci, and Pleiotropic Mechanisms across Eight Psychiatric Disorders. Cell 2019;179:1469-82.e11.

6. Muinos-Gimeno M, Guidi M, Kagerbauer B, et al. Allele variants in functional MicroRNA target sites of the neurotrophin-3 receptor gene (NTRK3) as susceptibility factors for anxiety disorders. Hum Mutat 2009;30:1062-71.

7. Pallanti S, Quercioli L. Treatment-refractory obsessive-compulsive disorder: methodological issues, 
operational definitions and therapeutic lines. Prog Neuropsychopharmacol Biol Psychiatry 2006;30:400-12.

8. Barcia JA, Reneses B, Nombela C. Precision surgery for obsessive compulsive disorder-which is the proper target? Ann Transl Med 2019;7:S184.

9. International Obsessive Compulsive Disorder Foundation Genetics C, Studies OCDCGA. Revealing the complex genetic architecture of obsessive-compulsive disorder using meta-analysis. Mol Psychiatry 2018;23:1181-8.

10. Schwarzenbach H. Methods for quantification and characterization of microRNAs in cell-free plasma/serum, normal exosomes and tumor-derived exosomes. Transl Cancer Res 2018;7:S253-63.

11. Flavell SW, Greenberg ME. Signaling mechanisms linking neuronal activity to gene expression and plasticity of the nervous system. Annu Rev Neurosci 2008;31:563-90.

12. Arora T, Bhowmik M, Khanam R, et al. Oxcarbazepine and fluoxetine protect against mouse models of obsessive compulsive disorder through modulation of cortical serotonin and CREB pathway. Behav Brain Res 2013;247:146-52.

13. Hall D, Dhilla A, Charalambous A, et al. Sequence variants of the brain-derived neurotrophic factor (BDNF) gene are strongly associated with obsessive-compulsive disorder. Am J Hum Genet 2003;73:370-6.

14. Qian Y, Song J, Ouyang Y, et al. Advances in Roles of miR132 in the Nervous System. Front Pharmacol 2017;8:770.

15. Vo N, Klein ME, Varlamova O, et al. A cAMP-response element binding protein-induced microRNA regulates neuronal morphogenesis. Proc Natl Acad Sci U S A 2005;102:16426-31.

16. Klein ME, Lioy DT, Ma L, et al. Homeostatic regulation of MeCP2 expression by a CREB-induced microRNA. Nat Neurosci 2007;10:1513-4.

17. Schratt GM, Tuebing F, Nigh EA, et al. A brain-specific microRNA regulates dendritic spine development. Nature 2006;439:283-9.

18. Liu W, Wu J, Huang J, et al. Electroacupuncture Regulates Hippocampal Synaptic Plasticity via miR-134Mediated LIMK1 Function in Rats with Ischemic Stroke.

Cite this article as: Yue J, Zhang B, Wang H, Hou X, Chen X, Cheng M, Wen S. Dysregulated plasma levels of miRNA-132 and miRNA-134 in patients with obsessive-compulsive disorder. Ann Transl Med 2020;8(16):996. doi: 10.21037/atm-20-5217
Neural Plast 2017;2017:9545646.

19. Gao J, Wang WY, Mao YW, et al. A novel pathway regulates memory and plasticity via SIRT1 and miR-134. Nature 2010;466:1105-9.

20. Privitera AP, Distefano R, Wefer HA, et al. OCDB: a database collecting genes, miRNAs and drugs for obsessive-compulsive disorder.Database (Oxford). 2015: bav069.

21. Frank DA, Greenberg ME. CREB: a mediator of long-term memory from mollusks to mammals. Cell 1994;79:5-8.

22. Kang H, Schuman EM. Long-lasting neurotrophininduced enhancement of synaptic transmission in the adult hippocampus. Science 1995;267:1658-62 .

23. Jeffery KJ, Reid IC. Modifiable neuronal connections: an overview for psychiatrists. Am J Psychiatry 1997;154:156-64.

24. Fan C, Zhu X, Song Q, et al. MiR-134 modulates chronic stress-induced structural plasticity and depression-like behaviors via downregulation of Limk1/cofilin signaling in rats. Neuropharmacology 2018;131:364-76.

25. Shen J, Xu L, Qu C, et al. Resveratrol prevents cognitive deficits induced by chronic unpredictable mild stress: Sirt1/miR-134 signalling pathway regulates CREB/BDNF expression in hippocampus in vivo and in vitro. Behav Brain Res 2018;349:1-7.

26. Lungu G, Stoica G, Ambrus A. MicroRNA profiling and the role of microRNA-132 in neurodegeneration using a rat model. Neurosci Lett 2013;553:153-8.

27. Nudelman AS, DiRocco DP, Lambert TJ, et al. Neuronal activity rapidly induces transcription of the CREBregulated microRNA-132, in vivo. Hippocampus 2010;20:492-8.

28. Peng J, Omran A, Ashhab MU, et al. Expression patterns of miR-124, miR-134, miR-132, and miR-21 in an immature rat model and children with mesial temporal lobe epilepsy. J Mol Neurosci 2013;50:291-7.

(English Language Editor: J. Reynolds) 